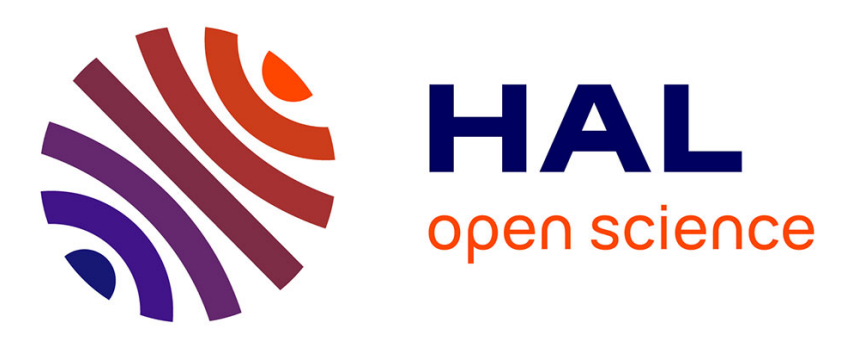

\title{
Assigning Codes in a Random Wireless Network
}

Fayçal Djerourou, Christian Lavault, Gabriel Paillard, Vlady Ravelomanana

\section{To cite this version:}

Fayçal Djerourou, Christian Lavault, Gabriel Paillard, Vlady Ravelomanana. Assigning Codes in a Random Wireless Network. 11th International Conference on Telecomunications, 2004, Fortaleza, Brazil. pp.348-353. hal-00004456

\section{HAL Id: hal-00004456 https://hal.science/hal-00004456}

Submitted on 14 Mar 2005

HAL is a multi-disciplinary open access archive for the deposit and dissemination of scientific research documents, whether they are published or not. The documents may come from teaching and research institutions in France or abroad, or from public or private research centers.
L'archive ouverte pluridisciplinaire HAL, est destinée au dépôt et à la diffusion de documents scientifiques de niveau recherche, publiés ou non, émanant des établissements d'enseignement et de recherche français ou étrangers, des laboratoires publics ou privés. 


\title{
Assigning Codes in a Random Wireless Network
}

\author{
Fayçal Djerourou, Christian Lavault, \\ Gabriel Paillard*, and Vlady Ravelomanana \\ LIPN, UMR CNRS 7030 \\ Université Paris XIII, 93430 Villetaneuse, France \\ \{fd, lavault, gap, vlad\}@lipn.univ-paris13.fr
}

\begin{abstract}
In this paper we present an algorithm that can assign codes in the Code Division Multiple Access (CDMA) framework for multihop ad hoc wireless networks. In CDMA framework, collisions are eliminated by assigning orthogonal codes to the stations such that the spectrum of frequency can be used by all transmitters of the network at the same time. In our setting, a large number $n$ of distinguishable stations (e.g. sensors) are randomly deployed in a given area of size $|S|$. We propose an efficient and fully distributed algorithm, which assigns codes to the nodes of our network so that, for any $\ell>0$, any two stations at distance at most $\sqrt{(1+\ell)|S| \log n / \pi n}$ from each other are assigned two distinct codes.
\end{abstract}

\section{Introduction}

Multihop ad hoc wireless networks, such as sensor networks are becoming a more and more important subject of research [7]. In this paper a network is a collection of transmitter-receiver devices, referred to as stations (processors or nodes). It is also assumed that each such station knows only its own identity (Id). Multihop wireless networks consist in a group of stations that can communicate with each other by messages over one wireless channel. Besides, messages may go through intermediate stations before reaching their final destination. At any given time $t$, the network may be modeled with its reachability graph: for any station $u$ and $v$, there exists one arc $u \rightarrow v$ iff $v$ can be reached from $u$.

The time is assumed to be slotted and in each time slot (round) every node can act either as a transmitter, or as receiver, but not both. At any given time slot, a station $u$ acting as a receiver gets a message if and only if exactly one of its neighbors transmits within the same round. If more than two neighbors of $u$ transmit simultaneously, $u$ is assumed to receive no message (collision). More precisely, the considered networks has no ability to distinguish between the lack of message and the occurrence of some collisions or conflicts. Therefore, it is highly desirable to design protocols working independently of the existence/absence of any collision detection mechanism. In this paper, we consider that a set of $n$ stations are initially randomly scattered (following the uniform distribution) in

\footnotetext{
* Supported by grant 1522/00-0 from CAPES, Ministry of Education, Brazil.
} 
a surface $\mathcal{S}$ of size $|\mathcal{S}|$. Typically, a global model for a mobile computing environment is a graph $G_{t}=\left(V, E_{t}\right)$ where $V$ is the set of the stations and $E_{t}$ is the set of links, which are present at time slot $t$. In our paper, the problem under consideration consists in minimizing the number of codes of the CDMA (Code Division Multiple Access), which is equivalent to coloring the graph $G_{t}[2]$. In the CDMA problem, collisions are removed by assigning orthogonal codes to the stations in such a way that the whole spectrum of frequency can be used by transmitters in the network at the same time. Each code designs the bit specification of each station in the network [1]. To deal with the CDMA, our algorithm solves the graph coloring problem.

\section{Coloring an Euclidean random network}

Our algorithm can be split in two steps as described in the following:

- First, each station has to discover its proper neighborhood. This is done using the randomized algorithm ExCHANGEID. This protocol needs $O\left((\log (n))^{2}\right)$ steps.

- Next, once the station nodes know their neighboorhood, we run AssignCODE which is a randomized (greedy) algorithm to assign codes to the current reachability graph.

\subsection{Discovering the neighborhood}

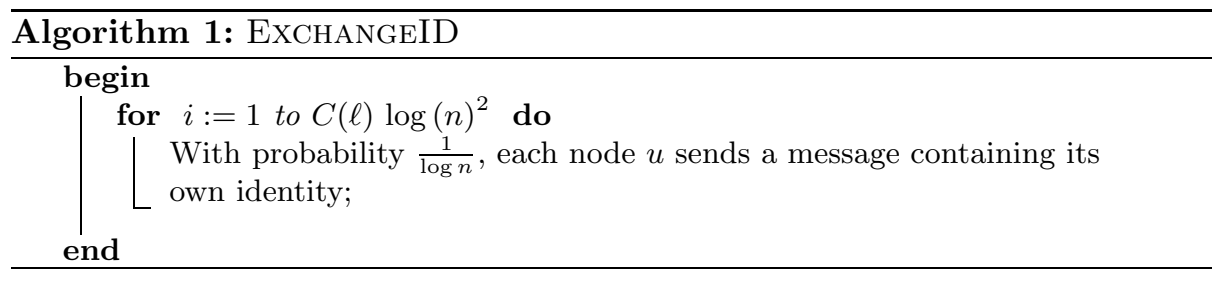

Theorem 1. For any fixed constant $\ell>0$, there exists a constant $C(\ell)$ such that if the transmission radius of each station is set to $r^{2}=\frac{(1+\ell)|\mathcal{S}|}{\pi n} \log n$, then, with probability tending to 1 as $n$ tends to $\infty$, every node receives all the identities of all its neighbors after an execution of EXCHANGEID.

Proof. The proof of Theorem 1 relies on the two following facts, viz., (i) the main properties of the random Euclidian network, and (ii) the number of iterations $T=C(\ell)(\log (n))^{2}$ in the loop of ExchANGEID is sufficient for each node to send its ID at least once to all its neighbors. For (i), we refer to the results of [8], which can be briefly stated as follows. Denote by $r$ the transmission range of the $n$ nodes randomly distributed in the surface $\mathcal{S}$ of size $|\mathcal{S}|=O(n)$. Then, in the following regimes, the graph is connected with high probability and we have

(a) For fixed values of $k$, that is $k=O(1)$, if $\pi \frac{n}{|\mathcal{S}|} r^{2}=\log n+k \log \log n+\omega(n)$, then the graph has asymptotically almost surely ${ }^{1}$ a minimum degree $\delta=k$.

\footnotetext{
${ }^{1}$ With probability tending to 1 as $n \rightarrow \infty$. For short, a.a.s. Here and throughout this paper $\omega(n)$ is a function tending to $\infty$ arbitrary slowly.
} 
(b) Let $k=k(n)$, but $1 \ll k(n) \ll \log n / \log \log n$.

If $\pi \frac{n}{|\mathcal{S}|} r^{2}=\log n+k(n) \log \log n$, then the minimum degree (resp. maximum degree) is a.a.s. $\delta=k(n)$ (resp. $\Delta=e \log n)$.

(c) If $\pi \frac{n}{|\mathcal{S}|} r^{2}=(1+\ell) \log n$ with $\ell>0$, then each node $v$ of the graph has a.a.s. $d_{v}$ neighbors, with

$$
-\frac{\ell \log n}{W_{-1}\left(-\frac{\ell}{e(1+\ell)}\right)}+o(\log n) \leq d_{v} \text { and } d_{v} \leq-\frac{\ell \log n}{W_{0}\left(-\frac{\ell}{e(1+\ell)}\right)}+o(\log n)
$$

where $W_{-1}$ and $W_{0}$ denote the two branches of the Lambert $\mathrm{W}$ function. We refer here to the paper of Corless et al. [3] for more precision on the Lambert W function, which is now a special mathematical function of its own (Note that it is implemented in almost all computer algebra tools such as MAPLE.)

Therefore, by inequality (1), within the regime considered in the assumptions of Theorem 1, the maximum degree of the graph is (with high probability) bounded by $c_{\ell} \log n$ (where $c_{\ell}$ satisfies, e.g. $c_{\ell}=2 W_{0}(-\ell / e(1+\ell)$ ) [3].

Using this latter remark, let us complete the proof of our Theorem. For any distinct pair $(i, j)$ of connected nodes, define $X_{i \rightarrow j}^{(t)}$ as follows: $X_{i \rightarrow j}^{(t)}=1$ iff the node $j$ does not receive the ID of $i$ at time $t \in\left[1,(\log (n))^{2} C(\ell)\right]$ and 0 otherwise.

In other terms, the set $\left\{X_{i \rightarrow j}^{(t)} \mid i, j \neq i, t \in\left[1,(\log (n))^{2} C(\ell)\right]\right\}$ is a set of random variables that counts the number of $\operatorname{arcs} i \rightarrow j$, such that $j$ never received the ID of $i$.

Denote by $X$ the r.v. $X=\sum_{i \neq j} X_{i \rightarrow j}$, where $X_{i \rightarrow j}=1$ iff $X_{i \rightarrow j}^{(t)}=1$ for all $t \in\left[1,(\log (n))^{2} C(\ell)\right]$. Now, we have the probability that $i$ does not succeed to send its ID to $j$ at time $t$ :

$$
\operatorname{Pr}\left[X_{i \rightarrow j}^{(t)}=1\right]=\left(1-\frac{1}{\log (n)}\right)+\frac{1}{\log n} \times\left(1-\left(1-\frac{1}{\log n}\right)^{d_{j}(t)}\right)
$$

where $d_{j}(t)$ denotes the degree of $j$ at time $t$.

Therefore, considering the whole range $\left[1,(\log (n))^{2} C(\ell)\right]$, after a bit of algebra we obtain $\operatorname{Pr}\left[X_{i \rightarrow j}=1\right] \leq\left(1-\frac{e^{-c_{\ell}}}{\log (n)}\right)^{\log (n)^{2} C(\ell)} \leq \exp \left(-\ln (n) e^{-c_{\ell}} C(\ell)\right)$, which bounds the probability that, for all $t \in\left[1,(\log (n))^{2} C(\ell)\right], i$ never sent its ID to $j$.

By linearity of expectations and since from (c) the number of edges is of or$\operatorname{der} O(n \log n)$, we then have $\mathbb{E}[X] \leq O\left(n \log (n) \exp \left(-\ln (n) e^{-c_{\ell}} C(\ell)\right)\right)$. Thus, $\mathbb{E}[X] \ll 1$ as $n \rightarrow \infty$ for a certain constant $C(\ell)$ such that, say $C(\ell) \geq 2 e^{c_{\ell}}$. (Note that this constant can be computed for any given $\ell$ by using, e.g. $c_{\ell}=2 W_{0}(-\ell / e(1+\ell))$ and this completes the proof of Theorem 1.) 


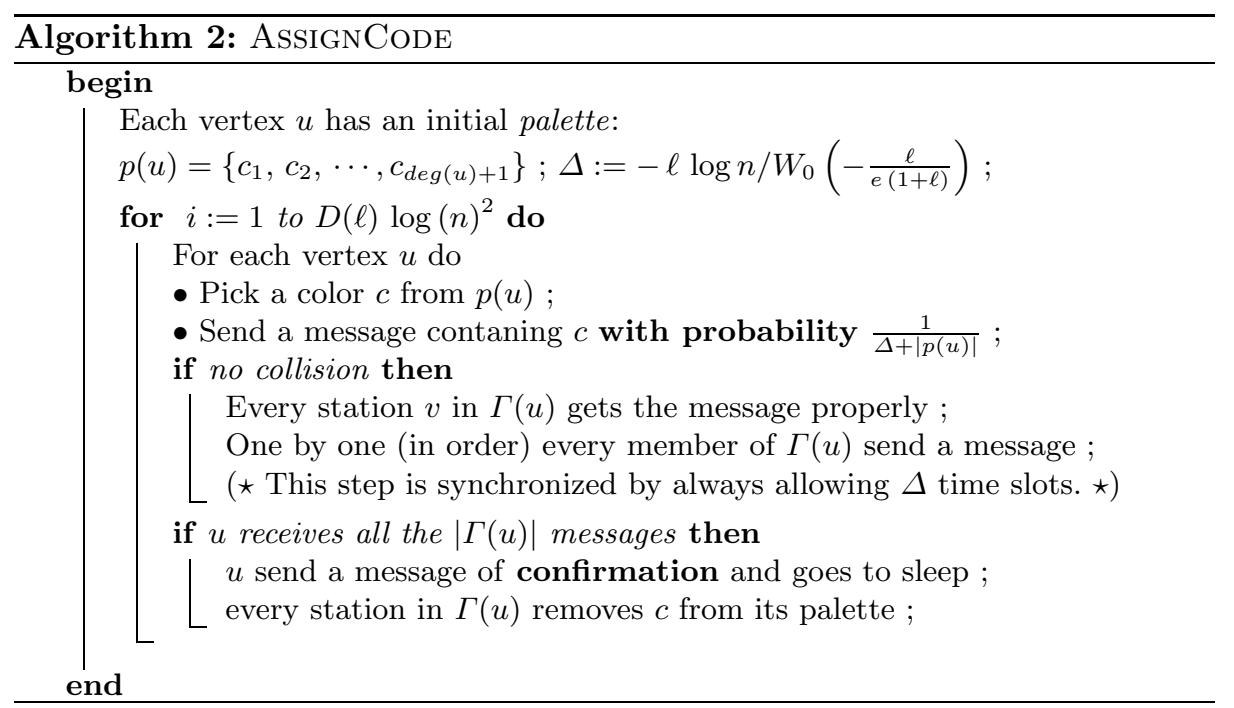

\subsection{Assigning codes}

To assign one code to each node of the network, we use the following protocol which we call AssignCoDE. Each vertex $u$ has an initial list of colors (palette) of size $\operatorname{deg}(u)+1$ and starts uncoloured. It is important to remark here that the stations know their neighbors (or at least a part of them) by using the previous algorithm, viz. ExCHANGEID. Then, the protocol AssignCoDE proceeds in rounds. In each round, each uncoloured vertex $u$, simultaneously and independently picks a color, say $c$, from its list. Next, the station $u$ attempts to send this information to his neighborhood denoted by $\Gamma(u)$. Trivially, this attempt succeeds iff there is no collision. Before its neighbors eventually assign the color $c$ to $u$, they has to send one by one a message of reception. Note that this can be done deterministically in time $O(\log n)$, since $u$ can attribute to its active neighbors in $\Gamma(u)$ a predefined ranking ranging from 1 to $|\Gamma(u)|$. Therefore, $u$ sends an aknowledgement message, and all its neighbors perform updates of their proper palettes and of their active neighbors. Hence, at the end of such a round the new colored vertex $u$ can quit the protocol.

Theorem 2. For any constant $\ell>0$, there exists a constant $D(\ell)$ such that if the transmission radius of each station is set to $r^{2}=\frac{(1+\ell)|\mathcal{S}|}{\pi n} \log n$, then, with probability tending to 1 as $n$ tends to $\infty$, any pair of nodes at distance at most $r$ from each other receives two different codes after invoking the protocol AssignCode.

Proof. Although more complicated, the proof of Theorem 2 is very similar to the one of Theorem 1 . For any distinct node $u$, recall that $\Gamma(u)$ represents the set of its neighbors and denote by $p_{u}$ the size of its current palette. Now, define the random variable $Y_{u}$ as follows: $Y_{u}=1$ iff the node $u$ remains uncoloured after the $D(\ell)(\log n)^{2}$ steps of AssignCODE and 0 otherwise. 
Denote by $\Gamma_{u}^{(t)}$ the set of active neighbors of $u$ at any given time $t$ during the execution of the algorithm. Suppose that we are in such time slot $t$. Independently of its previous attemps, $u$ remains uncoloured with probability

$$
p_{u, t}=\left(1-\frac{1}{\left(\Delta+p_{u}\right)}\right)+\frac{1}{\left(\Delta+p_{u}\right)} \times\left(1-\left(1-\frac{1}{\left(\Delta+p_{v}\right)}\right)^{\left|\Gamma_{u}^{(t)}\right|}\right) .
$$

There is at least a collision due to one neighbor $v \in \Gamma_{u}^{(t)}$.

Since $\forall t, \Gamma_{u}^{(t)} \leq \Delta$ and $\forall v, 1 \leq p_{v} \leq \Delta+1$, we have

$$
\begin{aligned}
p_{u, t} & \leq\left(1-\frac{1}{\left(\Delta+p_{u}\right)}\right)+\frac{1}{\left(\Delta+p_{u}\right)}\left(1-\left(1-\frac{1}{\Delta}\right)^{\left|\Gamma_{u}^{(t)}\right|}\right) \\
& \leq\left(1-\frac{1}{\left(\Delta+p_{u}\right)}\right)+\frac{1}{\left(\Delta+p_{u}\right)} \times\left(1-\left(1-\frac{1}{\Delta}\right)^{\Delta}\right) \\
& \leq 1-\frac{1}{e\left(\Delta+p_{u}\right)} \leq 1-\frac{1}{2 e \Delta} \leq 1-\frac{1}{6 \Delta} .
\end{aligned}
$$

Therefore, with probability at most $\left(1-\frac{1}{6 \Delta}\right)^{D(\ell) \log n^{2}} \leq \exp \left(-\frac{D(\ell) \log n^{2}}{6 \Delta}\right), u$ remains uncoloured during the whole algorithm. Thus, the expected number of uncoloured vertices at the end of the protocol AssignCode is less than $\mathbb{E}[Y]=$ $\sum_{u} \mathbb{E}\left[Y_{u}\right] \leq n \exp \left(-\frac{D(\ell) \log n^{2}}{6 \Delta}\right)$

Since by (1), we have $\Delta=\Delta(\ell) \leq-2 \frac{\ell \log n}{W_{0}\left(-\frac{\ell}{e(1+\ell)}\right)}$, it is now easy to choose a constant $D(\ell)$ such that $D(\ell)>-\frac{12 \ell}{W_{0}(-\ell / e(1+\ell))}$, and $\mathbb{E}[Y] \ll 1$ as $n \rightarrow \infty$. After using the well known Markov's inequality, the proof of our Theorem is completed.

\subsection{Efficiency of the algorithms}

Both protocols use local competitions, which means that the "coin flipping" games to access the shared wireless channel take place only between neighbors. First, we note that the lower bound for broadcasting in a network of diameter $D$ is given by $\Omega(D \log (n / D))$ [5]. A node $u$ in the network needs at least $O(|\Gamma(u)|)$ "local broadcasts". By "local broadcast" we mean the sending of information to nodes of distance at most $2 r$ where $r$ is the transmission range. From the main result of [5], it takes at least $\Omega(\log (\operatorname{deg}(u)))$ time slots to get all the IDs of the neighbors of $u$. By $(1),|\Gamma(u)|=\Theta(\log n)$, an algorithm needs at least $\Omega(\log n \times \log \log n)$ time slots to exchange the IDs of all the connected nodes. Therefore, our protocol ExCHANGEID, which needs $O\left((\log n)^{2}\right)$ time slots, is at most a $O(\log n / \log \log n)$ factor away from the optimal, and AssignCoDE is at most a $O(\log n / \log \log n)$ factor away from the optimal. 


\section{Final remarks}

This paper solves the problem of assigning different codes to stations randomly deployed in any given region $\mathcal{S}$. Our results make sense and can be useful for many reasons, including:

(i) Our settings is examplified for a large number $n$ of fire sensors dropped by planes in some large area $\mathcal{S}$. In our paper, the areas where such sensors are thrown need only be bounded. By contrast with numbers of existing papers, our results reflect real-life situations where the areas under consideration are far to be as regular as squares, rectangles or circles.

(ii) Our analysis yields key insights for the coloring problem in a rigorous framework, whereas a majority of results are based on empirical results.

(iii) Assume each deployed node to be "active" with constant probability $p$ $(0<p \leq 1)$. All our results can be extended by taking the intensity of the process $n /|\mathcal{S}|$ as $p n /|\mathcal{S}|$ (or by simply using $n^{\prime}=p n$ in the analysis). An "active" node is neither faulty nor asleep. This is especially well suited for "energy efficient" settings when some node are inative and saving their batteries, which increases the network's lifetime.

(iv) We considered herein uniform distributions, which can be approximated by Poisson point processes [4, pp 39-40]. Since Poisson processes are invariant [6] if their points are independently translated, the translations being identically distributed from some bivariate distribution (direction and distance), all our results remain valid. Therefore, the present results can serve to cope with mobile networks whenever the mobility model corresponds to the same translation distribution and whenever the $O\left((\log n)^{3}\right)$ time slots that are needed to achieve the coloring of the nodes can be neglected. Thus, the results of this paper can help both researchers and designers to face many realistic situations.

\section{References}

1. Battiti, R., Bertossi, A. A., Bonuccelli M. A.: Assigning Codes in Wireless Networks: Bounds and Scaling Properties. Wireless Networks, 5 (1999) 195-209

2. Bollobàs, B.: Modern graph theory. Graduate Texts in Mathematics. Springer, Berlin (1998)

3. Corless, R. M., Gonnet G. H., Hare D. E. G., Jeffrey D. J., Knuth D. E.: On the Lambert W Function. Advances in Computational Mathematics 5 (1996) 329-359

4. Hall, P.: Introduction to the Theory of Coverage Processes. Birkhäuser (1988)

5. Kushilevitz, E., Mansour, Y.: An $\Omega(D \log (N / D))$ Lower Bound for Broadcast in Radio Networks. SIAM Journal on Comput 27(3) (1998) 702-712

6. Miles, R. E.: On the Homogenous Planar Poisson Point Process. Math. Biosciences 6 (1970) 85-127

7. Perkins, C. E.: Ad Hoc Networking. Addison-Wesley (2001)

8. Ravelomanana, V.: Asymptotic Critical Ranges for Coverage Properties in Wireless Sensor Networks. In submitted, Available upon request (2003) 